\title{
Progreso genético mediante pruebas de rendimiento y de progenie en carneros Corriedale en la sierra central del Perú
}

\author{
Genetic progress through performance and progeny tests on Corriedale rams in \\ the central highlands
}
Fritz Carlos Trillo Zárate ${ }^{1,4}$, Cecilio Barrantes Campos ${ }^{1}$, Jimny Nuñez Delgado ${ }^{2}$, Nathalie Zirena Arana ${ }^{3}$

\section{Resumen}

\begin{abstract}
El objetivo del estudio fue comparar el progreso genético esperado de índices de selección para ovinos Corriedale en base a pruebas de rendimiento y progenie. El estudio se realizó en la Unidad de Producción «Corpacancha» de la SAIS Pachacútec, Junín, Perú, a una altitud de $4150 \mathrm{msnm}$. Se utilizó una prueba de rendimiento en función al peso vivo y peso de vellón, y modelo mixto para obtener el mejor predictor lineal insesgado (BLUP), donde los efectos fijos fueron sexo, tipo de parto y una covariable edad al destete. La progenie fue 118 hembras y 73 machos. El peso vivo a la primera esquila fue $65.86 \pm 13.25 \mathrm{~kg}$ y el peso de vellón limpio $5.99 \pm 1.44 \mathrm{~kg}$. La tasa de fertilidad fue $75 \%$, natalidad $109.7 \%$, partos mellizos $4.8 \%$ y 1.7 servicios/preñez. La correlación peso vivo con peso de vellón fue 0.47 , adecuada para los índices de selección. El progreso genético esperado del índice de selección por rendimiento fue 1920 y $132 \mathrm{~g}$ para peso vivo y peso del vellón a la primera esquila $\left(r^{2}=31.36\right)$, respectivamente. El índice de selección de la prueba de progenie mostró un progreso genético de $954.03 \mathrm{~g}\left(\mathrm{r}^{2}=40.54\right)$ para peso vivo y de $117.46 \mathrm{~g}\left(\mathrm{r}^{2}=40.49\right)$ para peso de vellón a la primera esquila. El índice de selección en base a pruebas de progenie da un progreso genético más confiable y los efectos fijos de sexo, tipo de parto y la covariable edad al destete ajustaron y sinceraron más el progreso genético de peso vivo.
\end{abstract}

Palabras clave: mejor predictor lineal insesgado; índice de selección; progreso genético; prueba de progenie

\footnotetext{
${ }^{1}$ Departamento de Producción Animal, Facultad de Zootecnia, Universidad Nacional Agraria la Molina, Lima, Perú

${ }^{2}$ Laboratorio de Ecología y Evaluación de Pastizales, Facultad de Zootecnia, Universidad Nacional Agraria la Molina, Lima, Perú

${ }^{3}$ Escuela de Medicina Veterinaria, Facultad de Ciencias Agropecuarias, Universidad Alas Peruanas, Lima, Perú

${ }^{4}$ E-mail: ftrillo@lamolina.edu.pe
}

Recibido: 18 de febrero de 2019

Aceptado para publicación: 28 de octubre de 2019 
The aim of this study was to compare the expected genetic progress of the selection indexes for Corriedale sheep based on performance and progeny tests. The study was carried out in the «Corpacancha» Production Unit of the SAIS Pachacútec, Junín, Peru, at an altitude of 4150 meters above sea level. A performance test was used based on bodyweight and fleece weight, and the mixed model to obtain the best unbiased linear predictor (BLUP), where the fixed effects were sex, type of parturition and a covariate age at weaning. The progeny was 118 females and 73 males. The bodyweight at first shearing was $65.86 \pm 13.25 \mathrm{~kg}$ and the clean fleece weight of $5.99 \pm 1.44 \mathrm{~kg}$. The fertility rate was $75 \%$, birth rate $109.7 \%$, twin births $4.8 \%$ and 1.7 services/pregnancy. The bodyweight correlation with fleece weight was 0.47 , suitable for the selection indices. The expected genetic progress of the performance selection index was 1920 and $132 \mathrm{~g}$ for bodyweight and fleece weight at first shearing $\left(r^{2}=31.36\right)$, respectively. The progeny test selection index showed a genetic progress of $954.03 \mathrm{~g}\left(\mathrm{r}^{2}=40.54\right)$ for bodyweight and $117.46 \mathrm{~g}\left(\mathrm{r}^{2}\right.$ $=40.49$ ) for fleece weight at first shearing. The selection index based on progeny tests gives more reliable genetic progress and the fixed effects of sex, type of delivery and the covariate age at weaning adjusted the genetic progress of bodyweight.

Key words: best unbiased linear predictor; selection index; genetic progress; progeny test

\section{INTRODUCCIÓN}

Los sistemas de producción extensivo de ovinos en el Perú tienen como objetivo de cría en lana obtener una calidad mediana, fina y superfina, mientras que en carne se usan razas carniceras o de doble propósito como la Corriedale, con base alimenticia de pastos cultivados (Ramírez y Rosario, 2007). Estos sistemas de producción manejan su propio programa de mejora genética, basándose en la selección visual como herramienta de elección de los mejores carneros. En vista de ello es necesario desarrollar índices de selección para obtener una mayor precisión en la selección de carneros tomando en cuenta los componentes ambientales y productivos (FAO, 2010).

En los programas de mejora genética se establece el objetivo de cría, el cual varía según el recurso genético, económico, natural, tecnológico y humano de los sistemas de producción animal (Müller et al., 2016). Para lograr los objetivos de cría, se deben desa- rrollar índices de selección que toman en cuenta valores genéticos, económicos y ambientales que pueden ser desarrollados con base a prueba de rendimiento y progenie (Cardellino, 2016). Existen varios métodos para estimar índices de selección, siendo el más usado actualmente el Mejor Predictor Lineal Insesgado (Best Linear Unbiased Prediction - BLUP), que tiene como ventaja analizar componentes ambientales y productivos. Además, estima el valor de cría en los programas de mejoramiento de las poblaciones animales (Legarra et al., 2005).

El empleo de índices de selección con base a pruebas de progenie permite obtener una buena predicción del valor de cría y de esa manera monitorear de manera precisa el progreso genético, ya que permite comparar animales de diferentes edades, sexos y tipos de parto (Giovannini et al., 2015). En tal sentido, el propósito del estudio fue comparar el progreso genético esperado de los índices de selección para ovinos Corriedale con base a pruebas de rendimiento y de progenie. 
Cuadro 1. Progenie de los carneros padres Corriedale evaluados según tipo de parto y sexo

\begin{tabular}{lccccc}
\hline \multirow{2}{*}{ Padre } & \multicolumn{2}{c}{ Tipo de parto } & \multicolumn{2}{c}{ Sexo } & \multirow{2}{*}{ Total } \\
\cline { 2 - 5 } & Simple & Doble & Machos & Hembras & \\
\hline 2A310 & 23 & 7 & 22 & 8 & 30 \\
2A312 & 5 & & & 5 & 5 \\
2A314 & 28 & 4 & 20 & 32 & 52 \\
IZ700 & 7 & & & 11 & 11 \\
IZ702 & 23 & 7 & 8 & 8 & 16 \\
IZ704 & 5 & & & 5 & 5 \\
AGMA66 & 29 & 6 & 2 & 15 & 17 \\
AGMA68 & 15 & & & 11 & 11 \\
AGMA70 & 20 & 7 & 21 & 8 & 29 \\
AGMA72 & 5 & & & 15 & 15 \\
\hline Total & 160 & 31 & 73 & 118 & 191 \\
\hline
\end{tabular}

\section{MATERIALES Y Métodos}

\section{Área del Estudio}

El estudio se realizó en la Unidad de Producción "Corpacancha» de la SAIS Pachacútec, ubicado en el distrito de Marcapomacocha, provincia de Yauli, departamento de Junín, Perú, a una altitud de 4150 $\mathrm{msnm}$. El hábitat corresponde a la región Puna, donde el clima es frio templado, con vastas extensiones de pastizales de porte alto apropiado para la crianza ovina. Los cambios estacionales son marcados, determinando la cantidad y calidad de las pasturas, al igual que la carga que soporta al pastorear los animales. La época de lluvias se inicia en noviembre y se prolonga hasta marzo (Tacuna et al., 2015).

\section{Animales}

Se utilizaron 10 carneros de raza Corriedale del plantel Cabaña, los que tuvieron una progenie de 118 hembras y 73 machos de clase Super (Cuadro 1). La evaluación se hizo a la primera esquila. Los carne- ros fueron de descendencia chilena con una buena alzada y característica bien definidas de la raza. La alimentación fue exclusivamente con pasto cultivado con una asociación trébol blanco (Trifolium repens L) y rye grass inglés (Lolium perenne L). El manejo del pastoreo fue rotacional, con pastoreo durante el día y recojo de los animales en dormideros en las noches. La reproducción fue asistida con inseminación artificial en todo el plantel.

\section{Mediciones en Campo}

Se tomó el peso vivo al nacimiento y al destete con un dinamómetro de $30 \mathrm{~kg}$ (50 g de error) colocado en un trípode. La sujeción del animal se hizo con un yute de $1.5 \times 1.0 \mathrm{~m}$ colocado por el pecho y vientre de la cría.

El peso de vellón y longitud de mecha se tomó a la primera esquila, 10 días después del destete, con una balanza digital $(10 \mathrm{~g}$ de error) y una regla de $30 \mathrm{~cm}$. La medición de la mecha se realizó en el costillar medio, luego se tomó una muestra de $50 \mathrm{~g}$ y en seguida se pesó el vellón. La muestra de fibra fue colocada en una bolsa de polietileno y trasla- 
dada al laboratorio del Programa de Ovinos y Camélidos Sudamericanos (POCA) de la Universidad Nacional Agraria la Molina (UNALM), en Lima.

\section{Mediciones en Laboratorio}

Se procedió al lavado de las muestras de lana. Para esto, se prepararon cuatro lavaderos con $40 \mathrm{~L}$ de agua $\mathrm{c} / \mathrm{u}$ a $50{ }^{\circ} \mathrm{C}$. El primero se usó para para sacar las impurezas, el segundo con $75 \mathrm{~g}$ de detergente no iónico alquil-fenol para retirar impurezas impregnadas fuertemente, el tercero con $25 \mathrm{ml}$ de jabón de carbonato sódico líquido para proporcionar suavidad y favorecer un mejor enjuague, y el cuarto para el enjuague definitivo (productos del Laboratorio Quimplex SA). Las muestras fueron secadas en un horno de 75-100 C y luego colocadas en una cámara con temperatura de $20^{\circ} \mathrm{C}$ y humedad de $70 \%$. Luego se procedió al cardado manual y, seguidamente, con una guillotina se tomó una muestra de $1 / 2$ pulgada de fibra que fue colocada en una placa Petri. La muestra se colocó en el fibrómetro Sirolan Laserscan TM, en solución de alcohol isopropílico a $18 \%$ de normalidad, procediéndose a la lectura de 1000 fibras efectivas. El fibrómetro usa la técnica de fotometría, calibrado según la norma IWTO-12 (IWTO-12, 2003) desarrollado por CSIRO de Australia (Quispe et al., 2018).

\section{Índice de Selección}

Para el desarrollo de la prueba de progenie se estimó el mejor predictor lineal insesgado (BLUP) utilizando el modelo macho, construyendo matrices de efectos fijos sexo y tipo de parto y la matriz de efectos aleatorios padres. Se usó la siguiente formula: $Y=a+X^{*} \beta+Z^{*} \alpha+\varepsilon$, donde $a$ es la covariable edad, $Y$ es el vector de producción, $\hat{a}$ es el efecto fijo (sexo tipo de parto), $X$ y $Z$ son las matrices de incidencia conocida relacionadas, $\alpha$ es el valor genético aditivo y $\varepsilon$ es el vector de residuales (Jhonson y Thompson, 1995).
Para la resolución de ecuaciones múltiples se empleó la siguiente matriz:

$$
\left[\begin{array}{cc}
X^{\prime} X & X^{\prime} Z \\
Z^{\prime} X & Z^{\prime} Z_{A^{-1}+\alpha}
\end{array}\right] \times\left[\begin{array}{l}
\beta \\
\delta
\end{array}\right]=\left[\begin{array}{l}
X^{\prime} Y \\
Z^{\prime} Y
\end{array}\right]
$$

\section{Valores Económicos}

Se utilizó la metodología de modelos bioeconómicos en ovinos propuesto por Müller et al. (2016), mediante las fórmulas: $a_{l}=\left(p_{1}\right)$ $(e+r s-1)$ y $a_{2}=\left(p_{2}\right)(r s-1) / 2$, donde $a_{1}$ y $a_{2}$ son los valores económico relativos $\mathrm{S} / . / \mathrm{kg}$ vellón y peso vivo a la primera esquila respectivamente, mientras $p_{1}$ y $p_{2}$ son los precios de mercado de peso de vellón y peso vivo, respectivamente, $e$ es las veces que se esquila un ovino en su vida productiva, $r$ es la tasa reproductiva del rebaño y $s$ es el número de servicios por preñez del rebaño. El programa R Statistic 3.4.1 se utilizó para resolver el sistema de ecuaciones matriciales en ambos índices y los parámetros de estos.

\section{Resultados y Discusión}

Los valores de índices productivos de plantel de la SAIS Pachacútec se muestran en el Cuadro 2, tanto para el rebaño como para los animales machos y hembras. El peso al destete fue tomado a una edad promedio de $126 \pm 21$ y $142 \pm 23$ días en hembras y machos, respectivamente. Franz et al. (2012) reportaron en ovinos Corriedale en pasturas naturales bajo un sistema extensivo, pesos al nacimiento de 3.8 y $3.9 \mathrm{~kg}$ y al destete de 22.40 y $23.4 \mathrm{~kg}$ en hembras y machos, respectivamente. Por otra parte, Díaz (2013), mencionan pesos de vellón en borregas de $2.7 \mathrm{~kg}$ y peso vivo de 76 y $45 \mathrm{~kg}$ en carneros y borregas, respectivamente. También, Freitas de Melo et al. (2019) indican peso vivo de borregas al primer parto de $49.8 \mathrm{~kg}$, corderos destetados de $16.5 \mathrm{~kg}$ y corderos nacidos de $5.2 \mathrm{~kg}$, bajo pasturas naturales en las pampas uruguayas. Estos resultados son inferiores a lo reportado en este estudio, pudiéndose de- 
Cuadro 2. Índices productivos del rebaño plantel ovino Corriedale de la SAIS Pachacútec

\begin{tabular}{lccc}
\hline Característica & Rebaño & Carneros & Borregas \\
\hline Peso vivo al nacimiento $(\mathrm{kg})$ & $4.28 \pm 0.81$ & $4.27 \pm 0.94$ & $4.28 \pm 0.73$ \\
Peso vivo al destete $(\mathrm{kg})$ & $34.78 \pm 7.26$ & $33.60 \pm 5.99$ & $35.44 \pm 7.8$. \\
Peso vivo a la primera esquila $(\mathrm{kg})$ & $65.86 \pm 13.25$ & $78.07 \pm 12.99$ & $60.01 \pm 9.8$ \\
Peso de vellón a la primera esquila $(\mathrm{kg})$ & $5.99 \pm 1.44$ & $7.06 \pm 1.48$ & $5.57 \pm 1.11$ \\
\hline
\end{tabular}

Cuadro 3. Correlaciones fenotípicas de características productivas en ovinos Corriedale de la SAIS Pachacútec

\begin{tabular}{cccc}
\hline Característica & PD & PVLL1 & PV1 \\
\hline PN & $0.33^{* *}$ & $0.52 * *$ & $0.24 * *$ \\
PVLL1 & & & $0.57 * *$ \\
\hline
\end{tabular}

PN: peso al nacimiento; PD: peso al destete; PVLL1: peso de vellón en la primera esquila; PV1: peso vivo en la primera esquila

$* *$ p value $(<0.01)$

Cuadro 4. Valores económicos relativos del peso vivo y de vellón de ovejas Corriedale de la SAIS Pachacútec

\begin{tabular}{lcc}
\hline Característica & $\begin{array}{c}\text { Precio } \\
\text { S./kg }\end{array}$ & $\begin{array}{c}\text { Ponderación } \\
\text { bioeconómica }^{1}\end{array}$ \\
\hline Peso vivo & 4.5 & 6.2 \\
Peso de vellón & 3.3 & 25.6 \\
\hline
\end{tabular}

${ }^{1}$ Adaptado de Müller et al. (2016)

ber a la alimentación exclusiva de pasto cultivado, permitiendo una mayor expresión del valor genético de los animales (Aliaga, 2006).

Los índices reproductivos fueron de $75 \%$ de fertilidad, $109.7 \%$ de natalidad, 1.7 servicios por preñez, $4.8 \%$ de partos de mellizos, $149.2 \pm 4.4$ días de gestación y 1.4 de corderos nacidos/madre. Estos valores son superiores a los obtenidos por un estudio si- milar de Barbato et al. (2011), quienes reportaron una fertilidad de $67.5 \%$ y natalidad $100.2 \%$, teniendo como pastura una asociación festuca (Festuca arundinacea Schreb) y trébol blanco (Trifolium repens L). Las tasas de fertilidad y natalidad en el presente estudio estuvieron dentro de los rangos esperados para un sistema de reproducción asistida de alto nivel productivo (Aliaga, 2006); posiblemente debido al manejo del flushing en las borregas antes del servicio y el sistema de alimentación basado en pasto cultivado.

Las correlaciones fenotípicas productivas utilizadas tanto para el índice de selección como otras adicionales se muestran en el Cuadro 3. Se encontraron evidencias que el peso al nacimiento está asociado con las características productivas de peso de vellón y peso vivo a la primera esquila en los ovinos Corriedale, por lo que esta característica podría ser utilizada para una selección temprana. También se demostró ${ }_{2}$ que el peso de vellón y peso vivo a la primera esquila están fuertemente asociados, como menciona

Cuadro 5. Heredabilidades y correlaciones genéticas de peso vivo y de vellón de ovejas Corriedale de la SAIS Pachacútec

\begin{tabular}{lcc}
\hline Característica & $\begin{array}{c}\text { Peso } \\
\text { vivo }\end{array}$ & $\begin{array}{c}\text { Peso de } \\
\text { vellón }\end{array}$ \\
\hline Peso vivo & 0.34 & 0.20 \\
Peso de vellón & & 0.36 \\
\hline $\begin{array}{l}\text { Fuente: Gimeno y } \\
\text { Sánchez et al. (2016) }\end{array}$ & &
\end{tabular}


Cuadro 6. Índices de selección en base a prueba de rendimiento y de progenie en carneros Corriedale de la SAIS Pachacútec

\begin{tabular}{lccc}
\hline Prueba & \multicolumn{1}{c}{ Índice } & $\begin{array}{c}\text { Progreso genético } \\
\text { PV1 }(\mathrm{g})\end{array}$ & $\begin{array}{c}\text { Progreso genético } \\
\text { PVLL1 }(\mathrm{g})\end{array}$ \\
\hline Rendimiento & $\mathrm{I}=2.03 *$ PV1+7.54*PVLL1 & 1920.00 & 132.00 \\
Progenie & $\mathrm{I}=5.92 *$ PV1+3.01*PVLL1 & 954.03 & 117.46 \\
\hline
\end{tabular}

PVLL1: peso de vellón en la primera esquila; PV1: peso vivo en la primera esquila

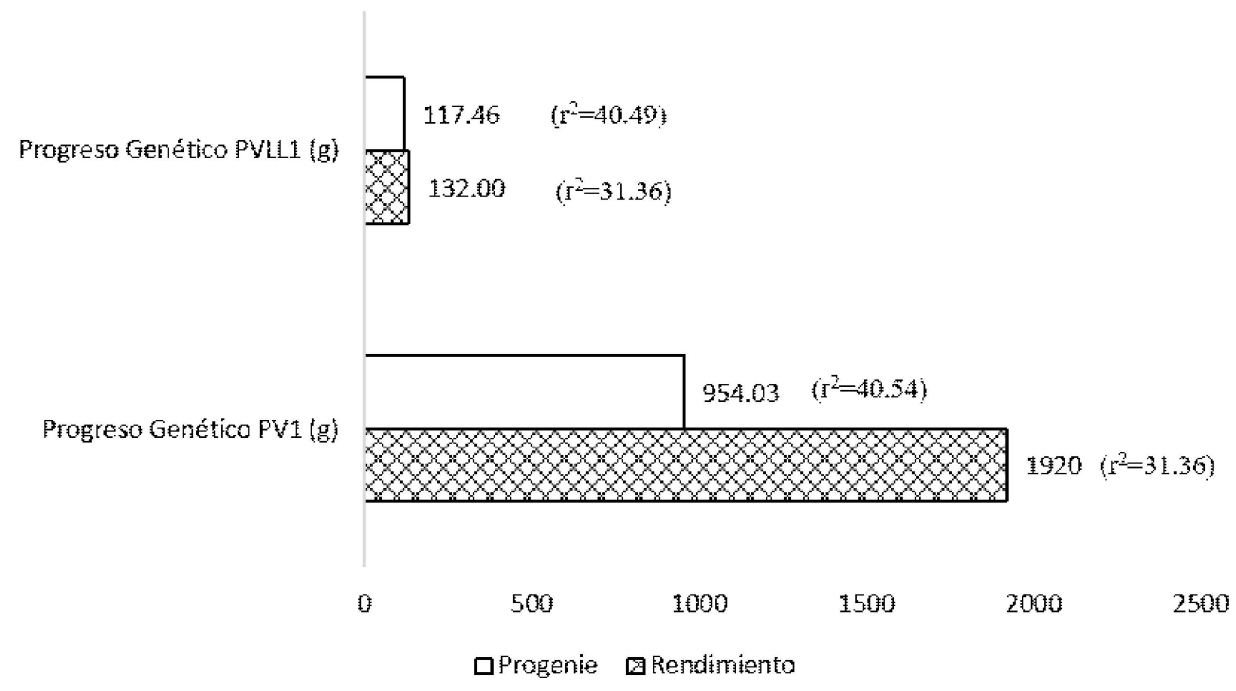

Figura 1. Comparación del progreso genético mediante índices de selección por rendimiento y prueba de progenie en ovinos Corriedale (SAIS Pachacútec, Perú)

Müller et al. (2016), de modo que ambas características pueden mejorar en forma conjunta en un proceso de selección.

Müller et al. (2016) propusieron modelos bioeconómicos basados en índices técnicos del rebaño y precios de mercado para hacer una ponderación bioeconómica, mientras que en este estudio se utilizaron precios referenciados en campo (Cuadro 4). Se determinó que el peso de vellón tiene mayor peso económico a pesar de su menor precio en el mercado, debido principalmente al nivel técnico de las SAIS Pachacútec.
Los parámetros genéticos utilizados para el desarrollo de los índices de selección fueron obtenidos de poblaciones con sistemas de crianza similares (Gimeno y Cardellino, 2006; Sánchez et al., 2016). Las heredabilidades fueron ajustadas a la variancia fenotípica de la población local como se muestra en el Cuadro 5. Los valores absolutos son los que interesan más que los relativos, si se divide el índice obtenido por una constante los valores absolutos no varían, pero se obtiene una ecuación que coincide casi en forma perfecta con un índice de selección en Merinos australianos (Müeller et al., 2016), criterio que debería tomarse en cuenta para 
discutir los índices de selección obtenidos. Lanari et al. (2012) establecen un índice con valores de ponderación para peso de vellón limpio de 17.030 y peso corporal de 0.924 en Corriedale argentino, y Barria et al. (1995) desarrollan un índice de selección donde el peso de vellón limpio se pondera en 17.02 y el peso corporal en 0.19 en Corriedale chileno. En ambos casos se da mayor ponderación al peso de vellón, pese que en ese entonces el mercado internacional no favorecía a la producción de lana.

Los resultados del presente estudio para el índice de selección estimado por prueba de rendimiento ponderan con 2.03 para peso vivo a la primera esquila y 7.54 para peso de vellón a la primera esquila; en tanto que el índice de selección por prueba de progenie pondera con 5.92 para peso vivo a la primera esquila y 3.02 para peso de vellón a la primera esquila (Cuadro 6). Las tendencias de ponderaciones en el índice de selección por prueba de rendimiento coinciden con las mencionadas por otros autores (Lembeye et al., 2014; Carrera et al., 2015). Por otro lado, se observó una menor ponderación en peso de vellón, debido a que el peso de vellón sucio y el precio de lana en el Perú es menor que en los mercados de Chile y Argentina. Mientras tanto, el índice de selección por prueba de progenie tiene una mayor ponderación al peso vivo a la primera esquila coincidiendo con el precio por kilogramo de mercado establecido.

La correlación del índice por prueba de rendimiento y el valor de cría resultante fue 0.56 , lo que indica un grado de asociación medio entre ambos valores. Carrera et al. (2015) desarrolló un índice de selección en ovinos Hampshire en la sierra central en función de peso vivo, peso al beneficio y ganancia de peso obtuvo correlaciones de $0.41 \mathrm{y}$ 0.53 , recomendando su aplicación en la selección de corderos. Sin embargo, Lembeye et al. (2014) en ovinos Merino en Chile utilizando peso de vellón sucio, diámetro de fibra, peso al nacimiento y tasa de crecimiento al destete obtuvo correlaciones de 0.92-0.93, siendo estos índices de selección muy eficientes para la selección. Con base a los resultados del presente estudio, se pueden recomendar índices de selección para rebaños con similares características de manejo.

Lanari et al. (2012) reportaron un progreso genético anual de $183 \mathrm{~g}$ en peso de vellón limpio y $2.90 \mathrm{~kg}$ en peso corporal, mientras Lembeye et al. (2014) reportaron en peso de vellón sucio de $100 \mathrm{~g}$. En el presente estudio, el progreso genético anual para el índice de selección por prueba de rendimiento fue $132 \mathrm{~g}\left(\mathrm{r}^{2}=31.36\right)$ en peso de vellón y de $1920 \mathrm{~g}\left(\mathrm{r}^{2}=31.36\right)$ en peso vivo a la primera esquila, mientras que en el índice de selección por prueba de progenie fue 117.46 $\mathrm{g}\left(\mathrm{r}^{2}=40.49\right)$ en peso de vellón y de $954.03 \mathrm{~g}$ $\left(r^{2}=40.54\right)$ en peso vivo a la primera esquila (Figura 1). Las diferencias con estos autores se atribuyen al empleo de peso de vellón sucio y la raza bajo evaluación. El progreso genético anual por prueba de progenie es el más preciso y queda demostrado en la primera generación filial.

\section{Conclusiones}

- El progreso genético esperado del índice de selección por rendimiento fue mayor en peso vivo a la primera esquila que el índice de selección de la prueba de progenie.

- El progreso genético de peso de vellón fue similar en ambos índices.

- El índice de selección en base a pruebas de progenie permite un progreso genético más confiable.

- El índice de selección por prueba de rendimiento se puede aplicar a características que no estén afectadas por efectos ambientales como el sexo y tipo de parto, siempre en cuando se manejen rebaños contemporáneos y bajo un mismo sistema de manejo. 


\section{Agradecimientos}

Los autores agradecen a la Sociedad Agrícola de Interés Social Pachacútec, y al Laboratorio de Ecología y Utilización de Pastizales (LEUP) de la Universidad Nacional Agraria La Molina por las facilidades prestadas para la realización de esta investigación.

\section{Literatura Citada}

1. Aliaga J. 2006. Producción de ovinos. Lima: Ed Gutemberg. 420 p.

2. Barbato G, Kremer R, Rosés L, Rista L. 2011. Producción de ovejas Corriedale y cruzas F1 con Milchschaf y Texel en condiciones de pastoreo. Veterinaria (Montevideo) 47: 9-13.

3. Barria N, Montaldo H, Jara A, Martinez V. 1995. Selection index construction for the Corriedale breed in Magallanes-Chile. In: Proc $6^{\text {th }}$ World Congress on Genetics Applied to Livestock Production. Armindale, Australia.

4. Cardellino R. 2016. Situación actual y perspectivas en el mercado de la lana. En: 10 años del Dohne Merino en Argentina. Trelew, Argentina. [Internet]. Disponible en: https://inta.gob.ar/sites/ default/files/cardellino_mercado_lanero_perspectivas.pdf

5. Carrera I, Chávez J, Meza E. 2015. Parámetros genéticos e índices de selección para corderos y borregas Hampshire bajo crianza intensiva en un rebaño de la costa central del Perú. Rev Inv Vet Perú 26: 66-76. doi: 10.15381/ rivep.v26i1.10908

6. Díaz R. 2013. Cadena productiva de ovinos. Lima, Perú. DPA MINAG. [Internet]. Disponible en: http:// agroaldia.minagri.gob.pe/biblioteca/ download/pdf/agroeconomia/ agroeconomia_ovino.pdf.
7. [FAO] Organización de las Naciones Unidas para la Alimentación y la Agricultura. 2010. Evaluación de las decisiones de inversión. En: Estrategias de mejora genética para la gestión sostenible de los recursos zoogenéticos. Roma: FAO. p 114-128.

8. Franz N, Aguilar DE, Vogel O, Flores AJ, Clavi M, Arias F, Rochinotti D. 2012. Mejora de la eficiencia productiva en ganadería vacuna-ovina. Proy. Reg. Ganadero. Cartera 20092012. EEA INTA Mercedes. [Internet]. Disponible en: https://inta.gob.ar/documentos/mejora-de-la-eficiencia-productiva-en-la-ganaderia-vacuna-y-ovina

9. Freitas De Melo A, Ungerfeld R, Pérez-Clariget R. 2019. Behavioral pattern in Texel x Corriedale terminal crossbreeding: maternal behavior score at birth, lambs' feeding behaviors, and behavioral responses of lambs to abrupt weaning. J Vet Behav 30: 9-15. doi: 10.1016/j.jveb.2018.10.007

10. Gimeno D, Cardellino RC. 2006. Genetic evaluation of sheep for wool and meat production in Uruguay. In: $8^{\text {th }}$ World Congress on Genetics Applied to Livestock Production. Belo Horizonte, MG, Brasil.

11. Giovannini N, Vozzi PA, Álvarez M, Maizon DO, Mueller JP. 2015. Genética. En: Mueller JP, Cueto MI, Robles CE (eds). Actualización en producción ovina. Bariloche, Argentina: INTA. p 95-111.

12. [IWTO-12] International Wool Textile Organization. 2003. Measurement of the mean and distribution of fibre diameter using the Sirolan-Laserscan fibre diameter analyser. In: IWTO Red Book Specifications. Ed 2015. Brussels: IWTO. $30 \mathrm{p}$

13. Jhonson DL, Thompson R. 1995. Restricted maximum likelihood estimation of variance components for univariate animal models using sparse matrix techniques and average information. $\mathrm{J}$ Dairy Sci 78: 449-456. doi: 10.3168/ jds.S0022-0302(95)76654-1 
14. Lanari MR, Reising C, Monzon M, Subiabre M, Killmeate R, Basualdo A, Cumilaf AM, Zubizarreta JL. 2012. Recuperación de la oveja Linca en la Patagonia. AICA 2: 151-154.

15. Legarra A, López-Romero P, Ugarte E. 2005. Bayesian model selection of contemporary groups for BLUP genetic evaluation in Latxa dairy sheep. Livestock Prod Sci 93: 205-212. doi: 10.1016/j.livprodsci.2004.10.008

16. Lembeye F, Castellaro G, Magofke JC, Uribe H. 2014. Comparación de distintos índices y criterios de selección en ovinos Merino Precoz manejados en condiciones extensivas de la zona central de Chile. Arch Med Vet 46: 389-397. doi: 10.4067/S0301-732X2014000300007

17. Müller J, Vozzi P, Giovannini N, Alvarez J. 2016. Beneficio del progreso genético en ovinos de la Argentina. Rev Invest Agropec 42: 307-316.
18. Quispe E, Sacchero D, Quispe M. 2018. Potencial uso en la evaluación de lanas y fibras de animales de un novedoso caracterizador electrónico. Rev Inv Vet Perú 29: 858-876. doi: 10.15381/ rivep.v29i3.13677

19. Ramírez D, Rosario I. 2007. Sectores ovinos en el Perú con perspectivas al 2015. En: V Congreso de Especialistas en Pequeños Rumiantes y Camélidos Sudamericanos. Mendoza, Argentina.

20. Sanchez AL, Urioste JI, Peñagaricano F, Neimaur K, Sienra I, Naya H, Kremer R. 2016. Genetic parameters of objectionable fibers and of their associations with fleece traits in Corriedale sheep. J Anim Sci 94: 13-20. doi: 10.2527/jas2015-9619

21. Tacuna R, Aguirre L, Flores E. 2015. Influencia de la revegetación con especies nativas y la incorporación de materia orgánica en la recuperación de pastizales degradados. Ecología Aplicada 14: 191-200. 